\title{
Susceptibilidad antimicrobiana de cepas de Escherichia coli obtenidas de muestras de heces de cerdos destinados a Consumo Humano
}

\author{
${ }^{1}$ Facultad de Medicina Veterinaria y Zootecnia - Universidad Peruana Cayetano Heredia \\ ${ }^{2}$ Facultad de Medicina Veterinaria - Universidad Nacional Mayor de San Marcos. \\ 3 Departamento Académico de Zootecnia - Universidad de Baja California Sur. México. \\ * enver.toledo@upch.pe \\ Aceptado para publicación: 01 de Octubre de 2015
}

Enver Toledo ${ }^{*}$, Néstor Falcon ${ }^{1}$, Cesar Flores², Mónica Rebatta ${ }^{2}$, José Guevara $^{3}$, Daphne Ramos²

\section{RESUMEN}

Escherichia coli es la especie bacteriana más común de la flora intestinal. La infección de cerdos por parte de cepas de E. coli es un problema frecuente, por lo tanto el conocimiento sobre la susceptibilidad antimicrobiana de estas cepas es importante, debido a que pueden llegar a infectar al ser humano produciendo enfermedades transmitidas por alimentos de difícil tratamiento. El objetivo del presente trabajo fue determinar la sensibilidad antimicrobiana de cepas de E. coli aisladas de cerdos aptos para consumo humano. Se tomaron en total de 40 muestras de heces de cerdos de las cuales se aislándose 36 cepas de E. coli. La evaluación antimicrobiana se realizó por el método de Kirby Bauer, para esta evaluación se consideraron 10 antibióticos de uso frecuente y algunos no autorizados para uso animal. Se encontró que el $100 \%$ de las cepas fueron resistentes a tetracicilina, el $97.2 \%$ a cefalotina, el $86.2 \%$ a ampicilina, el $77.8 \%$ a sulfametoxazol-trimetropin, el $75 \%$ para estreptomicina y el $63.9 \%$ a ácido nalidixico. La sensibilidad fue mayor para amikacina (88.9\%), gentamicina (80.6\%), ciprofloxacina (66.7\%), y cloranfenicol (55.6\%). El estudio evidencio la presencia de cepas de E. coli resistentes hasta a 8 antibióticos, lo que podría indicar un mal manejo de la terapia antimicrobiana durante la crianza de estos cerdos.

Palabras clave: E. coli, antimicrobianos, antibióticos, resistencia, cerdos

Antimicrobial susceptibility of Escherichia coli strains obtained from fecal samples from pigs for human consumption

\begin{abstract}
Escherichia coli is the most common bacterial species of the intestinal flora. Infection of pigs by strains of E. coli is a common problem, so the knowledge of antimicrobial susceptibility of these strains is important, because they can get to infect humans causing foodborne illness difficult to treat. The aim of this study was to determine the antimicrobial susceptibility of strains of E. coli isolated from pigs fit for human consumption. They took a total of 40 stool samples from pigs which 36 strains of E. coli is isolating. The antimicrobial evaluation was performed by the method of Kirby Bauer, 10 antibiotics for this assessment were considered often used and some are not approved for animal use. It was found that $100 \%$ of the strains were resistant to tetracycline, $97.2 \%$ to cephalothin, ampicillin $86.2 \%, 77.8 \%$ to trimethoprim-sulfamethoxazole, $75 \%$ to $63.9 \%$ streptomycin and nalidixic acid. The sensitivity was higher for amikacin $(88.9 \%)$, gentamicin $(80.6 \%)$, ciprofloxacin $(66.7 \%)$, and chloramphenicol $(55.6 \%)$. The study showed the presence of E. coli strains resistant to antibiotics 8 , which might indicate a misuse of antimicrobial therapy for raising these pigs. Keywords: E. coli, antibiotics, resistance, pigs.
\end{abstract}




\section{INTRODUCCIÓN}

La bacteria E. coli forma parte de la familia Enterobacteriaceae, conformada por 20 géneros, aproximadamente 120 especies y miles de serotipos. Está integrada por bacilos Gram negativos no esporulados, móviles con flagelos peritricos o inmóviles, aerobios-anaerobios facultativos (Starr, 1986). Esta bacteria es integrante de la flora normal del hombre y de muchos animales; por ello su presencia se considera como un indicador de contaminación fecal reciente (Lim, 2010).

Las cepas de E. coli se clasifican según los antígenos que poseen, siendo los principales el antígeno somático $(\mathrm{O})$, antígeno flagelar $(\mathrm{H})$ y el antígeno capsular (K) (Vadillo, 2002). En la actualidad se han diferenciado seis grupos de cepas patógenas que tienen diferente tipo de virulencia. Estos grupos son: la E. coli enteropatógena (EPEC), enterotoxigénica (ETEC), enterohemorrágica (EHEC), enteroinvasiva (EIEC), con adherencia difusa (DAEC) y enteroagregativa (EAEC). Cada uno de estos grupos presenta características distintas en cuanto a su epidemiología, patogénesis, manifestaciones clínicas y tratamiento (Levine, 1987).

E. coli es un patógeno entérico, el cual es la causa principal de diarrea en los países pobres. Se calcula que esta bacteria está produciendo la muerte de aproximadamente un millón de niños por año (Torres et al., 2001). Los síntomas ocurren cuando la bacteria coloniza por medio de sus pilis o fimbrias la mucosa del intestino delgado. Dentro de los cuadros clínicos reportados tenemos el síndrome urémico hemolítico producido por cepas productoras de toxina shiga, que ha sido relacionado con el consumo de carne molida (Vally et al., 2012); también puede producir diarrea aguda y acuosa, signo clínico categorizado como una de las principales causas de muerte en niños a nivel mundial (Arias et al., 2000). Asimismo, en recién nacidos se ha observado una diarrea liquida de color verde con moco y sin sangre; la cual puede llegar a persistir hasta 20 días (Eslava et al., 1994).

Diversos estudios indican que la mayor parte de animales destinados para consumo humano pueden ser reservorios de E. coli, dentro de ellos tenemos al cerdo el cual puede actuar como portador asintomático de la cepa $\mathrm{O} 157: \mathrm{H} 7$, que produce el síndrome urémico hemolítico en humanos (Hernández et al., 2008; Gallegos et al., 2009; Rahal et al., 2012). Esta bacteria puede adquirirse al consumir alimentos contaminados como carne, vegetales, frutas, etc., ya sean crudos o mal cocidos (Lim et al., 2010). Así tenemos, que la contaminación de la carne se puede producir en el proceso de sacrificio del animal, debido a que esta bacteria forma parte de la flora normal del intestino de los animales (Sánchez et al., 2011).

En la actualidad se conoce que el uso indiscriminado de antimicrobianos selecciona cepas resistentes a la terapia antibiótica; siendo este un fenómeno biológico/natural; el cual puede producirse por mutaciones en las bacterias o por la capacidad de estas de transferir horizontalmente su material genético, existiendo una clara correlación entre el uso de antibióticos y la resistencia bacteriana (OMS, 2001).

A nivel de animales de producción, se han realizado estudios para ver la susceptibilidad antimicrobiana. En un estudio se analizaron muestras de heces de crías de cerdos con diarrea y sin diarrea. Ellos encontraron tres tipos de Enterobacterias; siendo la más frecuente la E. coli; las cuales mostraron susceptibilidad ante la amikacina y multiresistencia frente a sulfametoxazol-trimetroprim, ampicilina, gentamicina, tetraciclina y cloranfenicol (Artiles et al., 2011). Otro estudio sobre aislamientos de E. coli de origen animal encontró que los patrones de sensibilidad y resistencia antibiótica resultaron variables en cuanto a la especie animal y el origen de los aislamientos, a excepción de la tetraciclina, donde en todos los grupos los porcentajes de resistencia estuvieron entre el 34 y 75\% (Carloni et al., 2011).

El uso de antimicrobianos en producción animal, ha tenido como objetivo principal tratar enfermedades que están comprometiendo la salud de los animales. En segunda instancia, los antimicrobianos se utilizan para prevenir la aparición de enfermedades; y en tercer lugar, para ayudar a mejorar la eficiencia nutricional de los animales en la forma de promotores de crecimiento. Por ello, el objetivo del estudio fue determinar el patrón de susceptibilidad antimicrobiana de la bacteria E. coli aislada de 
heces de cerdos destinados para consumo humano, utilizando como antimicrobianos los antibióticos que se aplican con frecuencia en la terapia humana y animal.

\section{MATERIALES Y METODOS}

Las muestras fueron tomadas de un centro de beneficio ubicado en la ciudad de Lima. Se recolectaron 40 muestras de heces de animales en buen estado de salud. El muestreo se realizó entre los meses de enero y mayo del 2013. De las 40 muestras tomadas se obtuvieron 36 colonias las que fueron enfrentadas a los antimicrobianos.

Para la determinación de cepas resistentes, se utilizó el método de discos de difusión en placa, también conocido como método de Kirby Bauer. Para la evaluación se utilizaron 10 antibióticos: amikacina (AMK), gentamicina (GEN), estreptomicina (EST), cefalotina (CEF), ciprofloxacina (CIP), sulfametoxazol-trimetropin (SXT), ampicilina (AMP), ácido nalidíxico (AN), cloranfenicol (CLO), tetraciclina (TET).

La lectura del antibiograma se realizó con la regla de Kirby Bauer. La categorización de las cepas se hizo en base al diámetro del área de inhibición alrededor del disco, de esta manera se estableció tres categorías: sensible, intermedio y resistente de acuerdo a tablas publicadas por el Comité Americano de Estandarización de Laboratorios Clínicos (NCCLS). Los resultados se resumieron en cuadros donde se puede observar la frecuencia según la categorización del halo de inhibición para los antibióticos utilizados en el estudio.

\section{RESULTADOS}

En el cuadro 1 se puede observar el número de cepas según el perfil de susceptibilidad o resistencia. La mayor cantidad de cepas fueron sensibles a los antibióticos amikacina y gentamicina y la totalidad fueron resistentes a tetraciclina. En el cuadro 2 se puede observar que la mayor cantidad de muestras fueron resistentes a 5 o más antibióticos.

\section{DISCUSION}

En la actualidad la resistencia antimicrobiana se ha incrementado a nivel mundial, habiéndose encontrado por ejemplo E. coli resistente a 2, 3 o 4 familias de antibióticos de relevancia clínica humana (Torres, 2012). Es conocido que existe una relación causa-efecto entre el uso de los agentes antimicrobianos y la aparición de resistencia frente a los mismos. En veterinaria el estudio de la aparición de resistencia se ha centrado en cepas de Salmonella typhimurium o bacterias entéricas como Escherichia coli. Por ser el intestino la localización principal del organismo donde tiene lugar la trasmisión de la resistencia (Ríos, 2004).

El estudio encontró que el $100 \%$ de las cepas aisladas de heces de cerdos aptos para consumo, fueron resistentes al menos a cuatro antibióticos de uso humano. Esto podría indicar que estos microorganismos estuvieron expuestos a estos antibióticos, se produjo una mutación en algún gen luego de la exposición al antibiótico o se produjo intercambio del material genético entre las bacterias (García y García, 1997).

Dentro de los antibióticos utilizados como promotores de crecimiento tenemos a la monensina, salinomicina, flavofosfolipol y avilamicina entre otros, los cuales actúan eliminado los microorganismos patógenos que se encuentran en el tracto gastrointestinal, aumentando así el área de absorción de nutrientes y por consiguiente mejorando la conversión alimenticia (Cancho et al., 2000).

En la crianza porcina se usan antibióticos como terapéuticos dentro de ellos tenemos la oxitetraciclina, la enrofloxacina, las cefalosporinas, la estreptomicina, las penicilinas, las sulfamidas y las ampicilinas (Moredo et al., 2007); todos estos antibióticos evidenciaron resistencia en el estudio. Asimismo, en cuadros entéricos los antibióticos más utilizados son la enrofloxacina, la estreptomicina, las cefalosporinas, el sulfametoxazol-trimetropim, la gentamicina y el florfenicol (Artiles et al., 2011). En este estudio se encontró que el $100 \%$ de las cepas aisladas fueron resistentes a la tetraciclina y a la oxitetraciclina. Esto tal vez se deba a que estos antibióticos comparten el mismo núcleo tetracícliconaftaceno, teniendo el espectro antimicrobiano, el mecanismo de acción y la toxicidad similar (Quintana et. al., 2009). En la especie porcina este antibiótico es usado para combatir las enfermedades respiratorias producidas 
Cuadro 1. Distribución Susceptibilidad antimicrobiana de cepas de Escherichia coli obtenidas de muestras de heces de cerdo destinados a beneficio

\begin{tabular}{lllllll}
\hline \multirow{2}{*}{ Antibiótico } & Sensible & & \multicolumn{2}{l}{ Intermedio } & \multicolumn{2}{l}{ Resistente } \\
\cline { 2 - 7 } & $\mathrm{N}^{\circ}$ & $\%$ & $\mathrm{~N}^{\circ}$ & $\%$ & $\mathrm{~N}^{\circ}$ & $\%$ \\
\hline AMK & 32 & 88,9 & 4 & 11,1 & -- & -- \\
GEN & 29 & 80,6 & 2 & 5,6 & 5 & 13,8 \\
EST & 2 & 5,6 & 7 & 19,4 & 27 & 75 \\
CEF & -- & -- & 1 & 2,8 & 35 & 97,2 \\
CIP & 24 & 66,7 & 5 & 13,9 & 7 & 19,4 \\
SXT & 7 & 19,4 & 1 & 2,8 & 28 & 77,8 \\
AMP & -- & -- & 5 & 13,8 & 31 & 86,2 \\
AN & 2 & 5,6 & 11 & 30,5 & 23 & 63,9 \\
CLO & 20 & 55,6 & 1 & 2,8 & 15 & 41,6 \\
TET & -- & -- & -- & -- & 36 & 100 \\
\hline
\end{tabular}

AMK: amikacina, GEN: gentamicina, EST: estreptomicina, CEF: cefalotina, CIP: ciprofloxacino, SXT: sulfametoxazol-trimetropim, AMP: ampicilina, AN: ácido nalidixico, CLO: cloranfenicol, TET: tetraciclina.

Cuadro 2. Muestras de cepas de Escherichia coli distribuidos según el número de antibióticos a los a los que son resistentes $(\mathrm{n}=36)$.

\begin{tabular}{lll}
\hline Número de antibióticos & \multicolumn{2}{c}{ Muestras resistentes } \\
\cline { 2 - 3 } Resistentes & Número & $\%$ \\
\hline Cuatro & 4 & 11.1 \\
Cinco & 9 & 25.0 \\
Seis & 7 & 19.4 \\
Siete & 6 & 16.7 \\
Ocho & 8 & 22.2 \\
Más de Ocho & 4 & 11.1 \\
\hline
\end{tabular}

por Pasteurella multocida o Mycoplasma spp. y también se usa en lechones destetados con gastroenteritis (Artiles et al., 2011; San Martín et al., 2005). 
La resistencia encontrada en este estudio para el caso del ácido nalidíxico puede relacionarse al uso de enrofloxacina (cuyo precursor es el ácido nalidixico) en granjas porcinas. Esta resistencia se podría deber a las mutaciones puntuales a nivel cromosómico o transferencia horizontal de material genético entre especies relacionadas o diferentes, facilitada por algunos elementos genéticos como los integrones del grupo I, que se caracterizan por captar genes que codifican determinantes de resistencia antibiótica (Mosquito et al., 2011). Siendo esta explicada por mutaciones en regiones determinadas de la DNA girasa y topoisomerasa IV (Sato et al., 2013).

Un estudio realizado en Cuba encontró una resistencia por encima del 50\% para sulfametoxazol trimetropim, ampicilina, gentamicina, teraciclina y cloranfenicol en cepas de cepas de E. coli aisladas de heces de crías de cerdos (Artiles et al., 2011). En el presente estudio se encontró similares resultados habiéndose determinado una resistencia del 77,8\% para sulfametoxazol trimetropim, 86,2\% a ampicilina y $100 \%$ para tetraciclina. En ambos estudios se encontró que las cepas eran sensibles para amikacina $(>80 \%)$.

La importancia de los resultados encontrados en este estudio se debe a que las cepas multiresistentes de E. coli puede llegar al humano a través de la carne, o a través de vegetales regados con agua contaminada con heces de animales o con el abono proveniente de estos animales considerados como portadores sanos de la bacteria (Falcón et al., 2010). La implementación de medidas de vigilancia sobre el uso de antibióticos en producción animal es necesaria para controlar la presencia de residuos antibióticos en productos de origen animal. El rol del médico veterinario es fundamental en los tratamientos de las diferentes patologías que afectan a los animales de producción, ya que los conocimientos le permiten decidir si es necesaria una terapia antibiótica.

\section{CONCLUSIONES}

Todas las cepas de E. coli aisladas de heces de cerdos mostraron resistencia antibiótica para tetraciclina y diferentes grados de resistencia para los otros antibióticos. Es necesario tomar las medidas para que se controle el uso de los antibióticos en la producción pecuaria.

\section{REFERENCIAS}

Arias, I., Cáceres, O., Salazar, E., Luz, M., \& Huguet R. (2000). Estudio de Escherichia coli enteropatogena y enteroagregativa en menores de cinco años de edad con diarrea persistente. Ministerio de Salud, Instituto Nacional de Salud. Informe Técnico 34.

Artiles, E., Arce, M., \& Mendoza, C. (2011). Estudio del comportamiento de la susceptibilidad antimicrobiana en aislados de heces de crías de cerdos en la Unidad Integral I de Palmira. REDVET. Revista Electrónica de Veterinaria, 12(3), 1-9.

Cancho, B., Garcia, M., Simal, J. (2000). El uso de antibióticos en la alimentación animal: Perspectiva actual. Cienci Tecnol Aliment, 3(1), 39-47.

Carloni, G., Pereyra, A., Denamiel, G., \& Gentilini, E. (2011). Resistencia a antimicrobianos en aislamientos de Escherichia coli de origen animal. In Vet, 13(2), 47-51.

Eslava, C., Mateo, J., \& Cravioto, A. (1994). Cepas de Escherichia coli relacionadas con la diarrea. diagnóstico de laboratorio de infecciones gastrointestinales. Giono S, Escobar A, Valdespino JL. Secretaria de Salud. México, 251.

Falcón, N., Ortega, C., Gorniak, S., Villamil, L. C., Ríos, C., \& Simón, M. C. (2010). El problema de la resistencia a antibióticos en salud pública. Una Salud, 1(1), 75-88.

Hernández, A., Ramos, A. Y., \& Hurtado, E. (2008). Presence of Escherichia coli, in raw pork chops sold retailed in the Maturín Municipality of Monagas State, Venezuela. Revista Cientifica UDO Agrícola, 8(1), 138-142.

Gallegos, M., Morales, A., Álvarez, G., Vásquez, J., Morales, L., Martínez, I., \& Maldonado, J. (2009). Caracterización de aislados de Escherichia coli O157: H7 en canales de bovinos y porcinos mediante PCR. Revista Cientifica, 19(2), 139-146.

García, R.J.A., \& García,S.E. (1997). Resistencias bacterianas $y$ antibioterapia. Eficacia in vivo 
Eficacia in vitro. Madrid-Barcelona: ed Doyma, SA, 39-50.

Levine, M. M. (1987). Escherichia coli that cause diarrhea: enterotoxigenic, enteropathogenic, enteroinvasive, enterohemorrhagic, and enteroadherent. Journal of infectious Diseases, 155(3), 377-389.

Lim, J. Y., Yoon, J. W., \& Hovde, C. J. (2010). A brief overview of Escherichia coli O157: H7 and its plasmid O157. Journal of microbiology and biotechnology, 20(1), 1-10.

Moredo, F. A., Vigo, G. B., Cappuccio, J. A., Piñeyro, P., Perfumo, C. J., \& Giacoboni, G. I. (2007). Resistencia a los antimicrobianos de aislamientos de Escherichia coli obtenidos de cerdos de la República Argentina. Revista argentina de microbiología, 39(4), 227-229.

Mosquito, S., Ruiz, J., Bauer, J. L., \& Ochoa, T. J. (2011). Mecanismos moleculares de resistencia antibiótica en Escherichia coli asociadas a diarrea. Rev Peru Med Exp Salud Publica, 28(4), 648-56.

Organización Mundial de la Salud. (2001). Estrategia mundial de la OMS para contener la resistencia a los antimicrobianos. Rev Panam Salud Pública, 10(4), 284-93.

Quintana, A., Medina, O., Nobrega, J,. Abreu, L., \& James, L. (2009). Determinación de residuos de tetraciclinas: oxitetraciclina, tetraciclina y clortetraciclina en tejido muscular de porcino mediante cromatografía líquida de alta resolución. Revista Facultad de Farmacia Venezuela, 72(1), 11-22.

Rahal, E. A., Kazzi, N., Nassar, F. J., \& Matar, G. M. (2012). Escherichia coli O157: H7-Clinical aspects and novel treatment approaches. Frontiers in cellular and infection microbiology, 2(138): 1-7 Ríos, I. A . (2004). Biodisponibilidad y metabolismo de un derivado fluorado del Tianfenicol en pollos Broiler (Doctoral dissertation, Universidad Complutense de Madrid).

San Martín, B., Bravo, V., \& Borie, C. (2005). Evaluación de la resistencia antimicrobiana en ganado bovino en Chile, utilizando E. coli como bacteria indicadora. Archivos de medicina veterinaria, 37(2), 117-123.

Sánchez de Ramos, M., de Díaz, C. G., \& Morán, A. E. (2011) Identificación de Escherichia coli O157: H7 en muestras de heces de pacientes con enfermedad diarreica aguda y en muestras de carne comercializadas en supermercados de San

Salvador, El Salvador. Minerva Revista en Línea CIC-UES, 2(1, 28-34

Sato, T., Yokota, S. I., Uchida, I., Okubo, T., Usui, M., Kusumoto, M., ... \& Tamura, Y. (2013). Fluoroquinolone resistance mechanisms in an Escherichia coli isolate, HUE1, without quinolone resistance-determining region mutations. Frontiers in microbiology, 4, 1-12

Starr, M. P. (1986). Edwards and Ewing's Identification of Enterobacteriaceae. By William H. Ewing. Elsevier Science Publishing Co., Inc., New York. 1986. 536 pages. ISBN 0-444-00981-7. International Journal of Systematic Bacteriology, 36(4), 581-582.

Torres, M. E., Pirez, M. C., Schelotto, F., Varela, G., Parodi, V., Allende, F., ... \& Ingold, E. (2001). Etiology of children's diarrhea in Montevideo, Uruguay: associated pathogens and unusual isolates. Journal of clinical microbiology, 39(6), 2134-2139.

Torres, C. (2012). La Resistencia Bacteriana a los Antibióticos, siete décadas después de Fleming. Academia de Farmacia, Zaragoza-España. 47 p. Vadillo, S., Yanes, E. M. M., \& Durán, S. P. (2002). Manual de microbiología veterinaria. McGraw-Hill Interamericana de España.

Vally, H., Hall, G., Dyda, A., Raupach, J., Knope, K., Combs, B., \& Desmarchelier, P. (2012). Epidemiology of Shiga toxin producing Escherichia coli in Australia, 2000-2010. BMC Public Health, $12(1), 63$. 